\title{
Gendered Personality Traits, Academic Programs and Academic Performance of Pakistani University Students: Evidence from Pakistan
}

\author{
Rafaquat Ali $^{\text {a }}$, Rabia Bahoo ${ }^{b}$, Bushra Shoukat ${ }^{c}$ \\ ${ }^{a}$ Assistant Professor, Department of Education, Bahawalnagar Campus, Bahawalnagar, The Islamia \\ University of Bahawalpur, Pakistan \\ Email: rafaquatiub@yahoo.com \\ ${ }^{\mathrm{b}}$ Lecturer, Department of Education, Government Sadiq College Women University, Bahawalpur, Pakistan \\ Email: rabia.bahoo@gscwu.edu.pk \\ ${ }^{c}$ Assistant Professor, Department of English, Bahawalnagar Campus, Bahawalnagar, The Islamia \\ University of Bahawalpur, Pakistan \\ Email: bushra.shoukat@iub.edu.pk
}

\begin{tabular}{l} 
ARTICLE DETAILS \\
\hline History: \\
Accepted 25 August 2021 \\
Available Online September 2021
\end{tabular}

\section{Keywords:}

Stereotype Gender Roles, Traditional Subject Choices, Stereotype Personality Traits, Academic Performance, Gendered Subject Choices

\section{JEL Classification: $P_{36}$}

DOI: $10.47067 /$ reads.v7i3.399

\begin{abstract}
The academic performance reflects students' success or failure to achieve learning objectives and goals in their academic endeavours. Previous studies have highlighted the major influence of students' gender, personalities, and academic programs on their academic performance. Different cultures, social settings, and educational systems promote different perceptions of gender roles that can produce different preferences of subjects and personality traits. Hence, the study aimed to assess the direct and total effects of Pakistani university students' gender, personality traits, and subject choices or academic programs on their academic performance. The Structure Equation Modeling approach was applied in two stages to find out the impacts of gender, personality traits, and academic programs on students' academic performance. The results showed that the gender variable is related to students' personality traits and the academic program. Although, gender did not have a direct impact on students' grades, but its total effect through personality traits and the academic program was significant. The personality traits were not found to have a direct or total significant effect on students' academic performance. The academic performances of students of some academic programs were higher as compared to students of other academic programs. The stereotype gendered personality traits and subject choices were present in students. The students' perceptions of stereotype gender roles, consequent personality traits, and subject choices should be intervened to improve the academic performance of both genders.
\end{abstract}

(C) 2021 The authors. Published by SPCRD Global Publishing. This is an open access article under the Creative Commons Attribution-

NonCommercial 4.0 


\section{Review of Economics and Development Studies, Vol. 7 (3) 2021, 433 - 451}

\section{Introduction}

Academic performance is critical because it defines success or failure in any course of study. It provides options for academic paths, and it limits employability in different professions and jobs. Therefore, teachers, parents, students, educational administrators, and education policymakers remain concerned about academic performance (Lamas, 2015). The academic performance is generally measured in terms of grades, and it describes students' academic attainments in any academic program in quantified form (Cachia et al., 2018; York et al., 2015). Hence, academic performance is the estimation of students' learning attainments as a result of his/her involvement in any instructional activity approved by an instructor (Lamas, 2015).

A large number of studies have underlined the roles of students' personal and contextual factors in their academic performance. Some important personal attributes that influence academic performance are students' gender (Hdii \& Fagroud, 2018), motivations, learning, and cognitive processing strategies, regulation of learning, learning orientation (Vermunt \& Vermetten, 2004), conceptions of learning, epistemology (Schommer-Aikins \& Easter, 2008), general intelligence (Kpolovie, 2017; Watson \& Monroe, 1990), emotional intelligence (MacCann et al., 2020) and personality (Andersen et al., 2020). Equally, some important contextual predictors of students' academic performance are socioeconomic background (Ali et al., 2019; Thomson, 2018), institutional environment (McDill et al., 1967), quality of instruction (Nortvedt et al., 2016; Weinert et al., 1989), and nature of the academic program (Ma, 2001; Shulrufa et al., 2010). To a considerable extent, these personal and contextual attributes of the academic performance vary in males and females (Pelch, 2018).

The literature draws attention to the role of gendered stereotypes in academic performance. The society, media, teachers, caregivers, and the implicit and explicit gendered curriculum of educational institutions propagate and encourage gendered social roles in students, and students acquire gendered stereotype behaviours (Gonzalez et al., 2020; Islam \& Asadullah, 2018). Consequently, these stereotypes determine their subject preferences and academic performances (Gonzalez et al., 2020). It means male and female students' academic performance is likely to differ in some subjects. The male students were found to have higher academic performance than female students in STEM subjects (Matz et al., 2017). Whereas female students have shown higher academic performance in language and humanities (Cavaglia et al., 2021; Hdii \& Fagroud, 2018). However, the difference in male and female academic performance in STEM subjects has shrunk, and there is consistently better performance of female students in language, arts, and humanities over the last two decades (Cavaglia et al., 2021; Hdii \& Fagroud, 2018). More than a decade ago, the Sparks-Wallace (2007) noticed that narrowness of differences in academic performance of male and female students has increased over the years. In this way, the academic performance of female students is improved in all subjects, regardless of their nature as scientific, technical, and languages (Hdii \& Fagroud, 2018).

Although, the gendered differences in academic performance have declined in recent years, many studies have documented the impact of students' personality traits on their academic performance. The student's academic performance relates to their different personality traits (Poropat, 2009). There is mounting evidence that the Neuroticism trait has negative implications on students' academic performance (Komarraju et al., 2011). Whereas personality traits of Conscientiousness, Agreeableness, and Openness to experience are linked with students' better academic performance (Poropat, 2009). There is inconclusive evidence about the Extraversion trait's positive role in better academic performance (O'Connor \& Paunonen, 2007). Although the importance of personality traits in academic performance is conclusive, the notion of gendered personality traits is present in literature 
Review of Economics and Development Studies, Vol. 7 (3) 2021, 433 - 451

(Weisberg et al., 2011; Yousaf Zai \& Jan, 2019). The literature leads to conclude that women might have higher levels of Agreeableness, Openness to experience, and Neuroticism (Chapman et al., 2007; Rahmani \& Lavasani, 2012). However, there is inconsistent evidence about gender differences in Extraversion, and Conscientiousness traits (Costa Jr. et al., 2001; Kim et al., 2016; Rahmani \& Lavasani, 2012; Yousaf Zai \& Jan, 2019). The perceived gendered social and occupational roles generate gendered personality traits (Costa Jr. et al., 2001).

Although the gender differences in academic performance in STEM subjects have decreased in the recent decade, the stereotype gendered subject choices persist (Gasteen, 2019). Although females have started to enrol in traditional masculine subjects in recent years (UNECE, 2019), males are more likely to take STEM subjects at the university level than females in OECD countries (Cavaglia et al., 2021). The female students may be overrepresented in health and cultural subjects, while male students may be overrepresented in science and economy subjects (Korpershoek et al., 2012). Additionally, there are gendered preferences within science subjects. The females prefer to study biology as compared to males who prefer to study physics (Gasteen, 2019).

It is worthwhile to mention that these stereotype subject choices and stereotype attitudes are stronger in males as compared to females. Females have started to take interest in the traditional masculine subject, but males do not show interest in traditional feminine subjects (van der Vleuten et al., 2016; Whitehead, 1996). Previous studies suggest that gendered patterns in the choices of subjects are the results of social conditioning and gender-biased environments (Gasteen, 2019). The field of science and science occupations are socially perceived as not suitable for females (Dom \& Yi, 2018; van der Vleuten et al., 2016). Subsequently, females opt to enrol in non-science subjects more than in science subjects (Dom \& Yi, 2018). Nowadays, the underrepresentation of females in STEM subjects at the university level is a challenge for modern societies and education systems (Cavaglia et al., 2021).

The results of several studies have established that gendered personality traits produce gendered subject choices (Korpershoek et al., 2012), and the personality traits mediate relationships between gender and subject choices (Vedel, 2016). Therefore, personality traits may predict students' interests in different subjects (Balsamo et al., 2012). Vedel (2016) found that higher Neuroticism, Agreeableness, and Openness traits are linked to choosing arts/humanities and psychology subjects, whereas higher Openness and Extraversion traits are associated to opt for law, political science, and economics subjects. Likewise, the higher Extraversion trait may not be associated with the choice of science subjects (Korpershoek et al., 2012).

\section{Conceptual Framework}

Conclusively, personal factors such as gender, personality, and contextual factors such as subject choices impact students' academic performance. These factors have direct and indirect impacts on students' academic performance. The factor gender can impact students' personality traits, subject choices, and academic grades. However, the social and cultural factors foster gendered roles, gendered competency beliefs, and gendered perceptions of occupations. Therefore, the different social and cultural setups may endorse different gendered personality traits, subject choices, and academic performance. Therefore, it is pertinent to study the total and direct effects of students' personal and contextual factors on academic performance in a single study because these can delimit academic performance (Crowther \& Briant, 2021; Lamas, 2015). Hence, the current study explained the impacts of students' gender, personality traits, and academic programs on their academic performance. The following conceptual framework was applied in this study. 


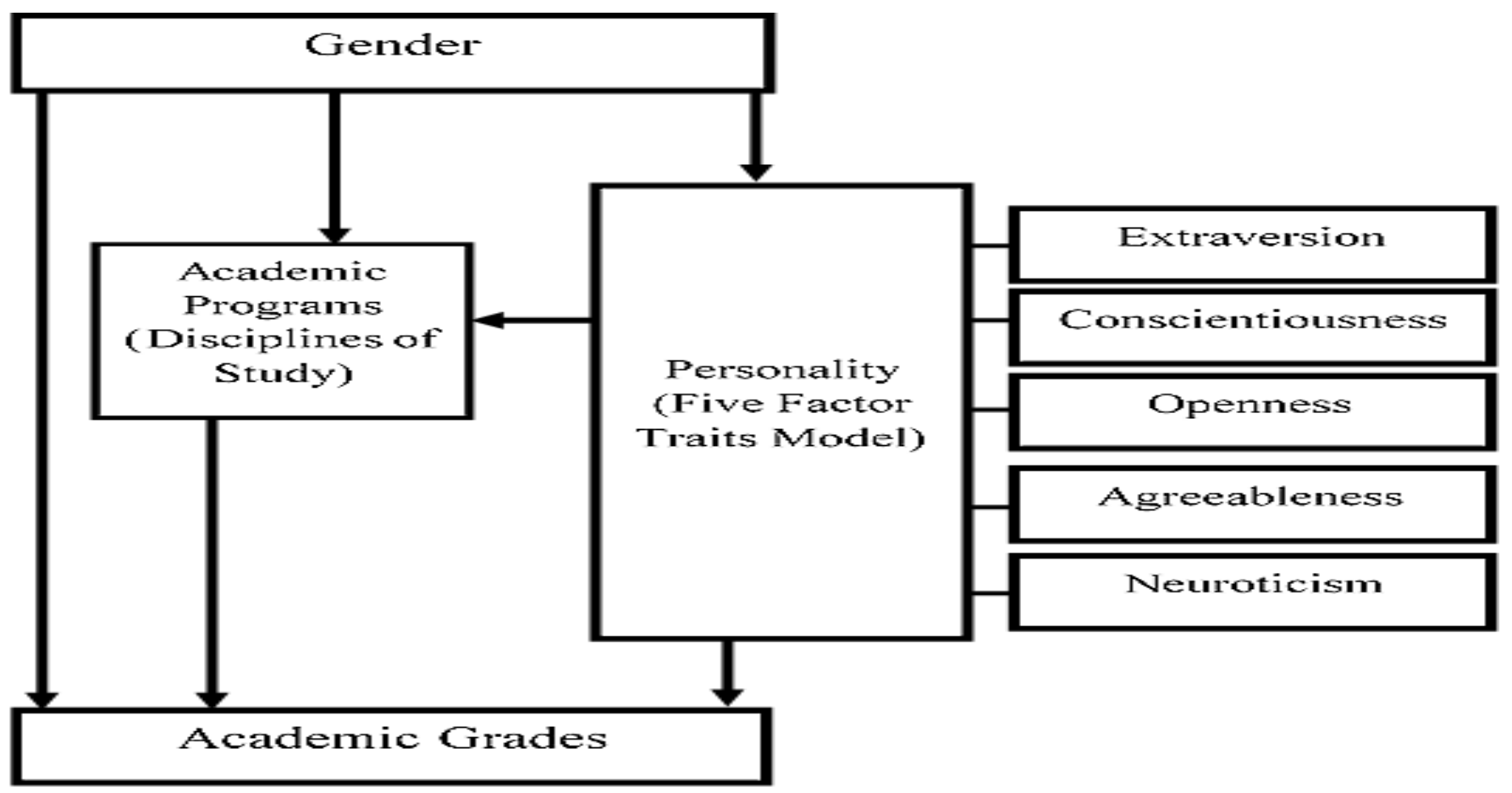

\section{Figure 1: Conceptual Framework}

According to the above conceptual framework, the current study envisioned direct and total effects of students' gender, personality traits, and academic programs on their academic performance. Subsequently, the objective of the study is to find out the effects of students' gender, personality traits, and academic programs (Major Subjects of study) on their academic performance. Following postulates were formulated to achieve the objectives of the study:

Hypothesis I: The students' gender, personality traits, and academic programs (Major Subjects of study) will have no significant direct impact on their academic performance.

Hypothesis II: The students' gender, personality traits, and academic programs (Major Subjects of study) will have no significant total effect on their academic performance.

\section{Method}

\subsection{Sample}

The participants in this study were students from different departments of the Islamia University of Bahawalpur, Bahawalnagar campus. This campus is located in the Bahawalnagar city in southern Punjab. The literacy rate in the Bahawalnagar district (50.3\%) is far below the literacy rate of Punjab (64\%) (PSLM, 2021). Also, the education score (14.4) of the Bahawalnagar district is below than the average score (17.26) of all districts in Punjab (PSLM, 2021). A total of 280 students voluntarily participated in this study. Among these respondents, 192 were female and 88 were male. The age of 96 percent of respondents was in the range of 18 years to 25 years. A total of 171 students stated their residential status, and among these, 56 percent of students were from a rural background and 44 percent were from an urban background. 


\section{Review of Economics and Development Studies, Vol. 7 (3) 2021, 433 - 451}

\subsection{Data Collection}

The students filled online Google form to provide their demographic information such as gender, academic program, and academic performance. The personality measures comprised different items freely available on International Personality Item Pool (IPIP) website. The students pointed out their agreement with statements of items to express their personality traits. The previous studies have acknowledged that these items, sub-traits, and traits have adequate reliability and validity (Johnson, 2014). The 28 items were selected from the IPIP pool to measure 05 personality traits. These five personality traits have sub-traits. The Openness trait has sub-traits emotionality, intellect, and artistic interests, and the Conscientiousness trait consists of cautiousness, self-discipline, and achievement striving sub-traits. Whereas the trait Extraversion included assertiveness, friendliness, and excitement sub-traits. Likewise, trust, altruism, and sympathy were the sub-traits of the Agreeableness trait, and the Neuroticism personality trait was limited to sub-traits of anxiety, depression, and anger. The items of these personality sub-traits have a five-point scale, which comprised options ranging from $1=$ very inaccurate, $2=$ moderately inaccurate, $3=$ neither accurate nor inaccurate, $4=$ accurate, and $5=$ very accurate. The reversed scores were assigned to negative statements.

\subsection{Data Analysis}

The current study involved hierarchical constructs. Therefore, the use of SmartPLS2 was logical because the PLS-SEM has been proven a good choice to comprehend the bigger picture that incorporates hierarchal latent variables interconnected in complex layered models (Akter et al., 2017). Furthermore, the PLS-SEM approach is useful because of its robustness to small sample size, normality issues of data, easiness to use measurement and structural modeling, and being an exploratory approach of research (Ringle et al., 2012). In hierarchical second-order modeling, the common approach used in PLS-SEM is the two-step approach (Anderson \& Gerbing, 1988). In the first step, the measurement model is tested to determine the reliability, convergent validity, and discriminant validity of the dimensions and associated items (Thien, 2020). In the second step, the scores of latent variables are used to formulate second-order constructs for modeling (van Riel et al., 2017) and the path relationships of second-order latent variables are determined (Tehseen et al., 2017). In this way, the first-order interrelated variables are channelled into a less, and manageable number of second-order variables to provide a simpler, and a more understandable version of the hierarchical model (Roni et al., 2015). Generally, the researchers prefer a two-step approach when they are more interested in paths and their significance from and to second-order constructs (Becker et al., 2017). Following the popular approach in hierarchical modeling, data analysis in this study comprised two stages. The first stage analysis consisted of measurement model analysis. In this stage, the objective was to identify the adequacy of the measurement model for all variables in the model. Based on the measurement model, the scores of second-order latent variables were calculated, and later on, these scores were used to test postulated paths in second-order analysis. The second stage analysis involved the testing of paths between second-order personality variables (Personality Traits) and the other variables in the model.

\section{Results}

The data analysis results are presented in two sections. The first section consists of the results of measurement model analysis in the first stage analysis. Section two consists of the result of secondstage analysis that covers structural modelling of second-order latent variables. Figure No.2 shows the results of two sections combined manually for the understanding of the reader.

\subsection{Measurement Model Analysis (First Stage Analysis)}

In the measurement model the variables gender, academic programs, and academic performance were not hierarchical latent variables and these were used in second stage analysis. However, the 
Review of Economics and Development Studies, Vol. 7 (3) 2021, 433 - 451

personality construct has hierarchal latent variables. The measurement model results of first stage analysis show that 28 items for 15 Personality sub-traits were found adequate and valid in this study (Figure No.2 and Table No.1). The 15 sub-traits (first-order latent variables) were minimized into five personality traits (Second-order latent variable). The different indicators in the measurement model have adequate loadings on the latent variable. The composite reliability and average variance extracted depicted the adequacy of the measurement model. All latent variables in this measurement model have AVE values above 0.5, and the composite reliability above 0.7 (Hair et al., 2019). With an exception, item loadings on cautiousness sub-trait were above 0.5 , but insignificant. However, these items were retained in the cautiousness sub-trait because these have item loadings greater than 0.5 , though these were insignificant (Hair et al., 2014). The items of the remaining sub-traits have item loadings significant and above 0.5 , and consequently, these items were retained in the measurement model (Hair et al., 2014). The final measurement model to measure students' personality traits has the following validity and reliability indices.

\begin{tabular}{|c|c|c|c|c|c|c|}
\hline \multicolumn{7}{|l|}{ Table No.1 } \\
\hline \multicolumn{7}{|c|}{ Item Loadings, Reliability, and Convergent Validity } \\
\hline $\begin{array}{l}\text { Personality } \\
\text { Trait }\end{array}$ & $\begin{array}{l}\text { Personality } \\
\text { Sub-trait }\end{array}$ & Items & $\begin{array}{l}\text { Item } \\
\text { Loadings }\end{array}$ & T Statistics & $\begin{array}{l}\text { Composite } \\
\text { Reliability }\end{array}$ & AVE \\
\hline \multirow[t]{5}{*}{ Agreeableness } & \multirow[t]{2}{*}{ Altruism } & ALTR-3 & 0.831 & $35.836^{* * *}$ & \multirow[t]{2}{*}{0.816} & \multirow[t]{2}{*}{0.690} \\
\hline & & ALTR-4 & 0.830 & $38.927^{* * *}$ & & \\
\hline & \multirow[t]{2}{*}{ Sympathy } & SYMP-3 & 0.740 & $13.776^{* * *}$ & \multirow[t]{2}{*}{0.748} & \multirow[t]{2}{*}{0.597} \\
\hline & & SYMP-4 & 0.804 & $19.481^{* * *}$ & & \\
\hline & Trust & Trus-3 & 1.000 & & 1.000 & 1.000 \\
\hline \multirow{6}{*}{$\begin{array}{l}\text { Conscientious- } \\
\text { ness }\end{array}$} & \multirow{2}{*}{$\begin{array}{l}\text { Achievement- } \\
\text { Striving }\end{array}$} & Achi-1 & 0.727 & $9.030^{* * *}$ & \multirow[t]{2}{*}{0.764} & \multirow[t]{2}{*}{0.619} \\
\hline & & Achi-2 & 0.843 & $17.866 * * *$ & & \\
\hline & \multirow[t]{2}{*}{ Cautiousness } & CAUT-1 & 0.796 & $1.395^{\text {N.S }}$ & \multirow[t]{2}{*}{0.804} & \multirow[t]{2}{*}{0.673} \\
\hline & & CAUT- 4 & 0.844 & $1.608^{\text {N.S }}$ & & \\
\hline & \multirow[t]{2}{*}{ Self-discipline } & Selfd-1 & 0.771 & $15.550^{* * *}$ & \multirow[t]{2}{*}{0.770} & \multirow[t]{2}{*}{0.627} \\
\hline & & Selfd-2 & 0.811 & $23 \cdot 347^{* * *}$ & & \\
\hline \multirow[t]{6}{*}{ Neuroticism } & \multirow[t]{2}{*}{ Anger } & Anger-1 & 0.828 & $24.388^{* * *}$ & \multirow[t]{2}{*}{0.811} & \multirow[t]{2}{*}{0.682} \\
\hline & & Anger-2 & 0.823 & $21.154^{* * *}$ & & \\
\hline & \multirow[t]{2}{*}{ Anxiety } & Anx-2 & 0.666 & $7.177^{* * *}$ & \multirow[t]{2}{*}{0.758} & \multirow[t]{2}{*}{0.615} \\
\hline & & Anx-3 & 0.887 & $22.761 * * *$ & & \\
\hline & \multirow[t]{2}{*}{ Depression } & Depr-1 & 0.882 & $45.281 * * *$ & \multirow[t]{2}{*}{0.826} & \multirow[t]{2}{*}{0.704} \\
\hline & & Depr-3 & 0.794 & $17.470^{* * *}$ & & \\
\hline \multirow[t]{5}{*}{ Openness } & Artistic & Art-in-1 & 0.589 & $4.409^{* * *}$ & 0.718 & 0.571 \\
\hline & Interest & Art-in-2 & 0.891 & $19.820 * * *$ & & \\
\hline & Intellect & INT-1 & 1.000 & & 1.000 & 1.000 \\
\hline & Emotionality & Emot-1 & 0.704 & $7.484^{* * *}$ & 0.749 & 0.601 \\
\hline & & Emot-2 & 0.840 & $18.444^{* * *}$ & & \\
\hline Extraversion & Assertiveness & Assr-2 & 0.855 & $44.717^{* * *}$ & 0.829 & 0.708 \\
\hline & & Assr-3 & 0.827 & $26.445^{\star * *}$ & & \\
\hline & Excitement & Excs-1 & 0.921 & $29.222^{* * *}$ & 0.758 & 0.619 \\
\hline & seeking & Excs-2 & 0.625 & $5.097^{* * *}$ & & \\
\hline & Friendliness & Fris-1 & 0.717 & $8.427^{* * *}$ & 0.750 & 0.601 \\
\hline & & Fris-2 & 0.829 & $14.916 * * *$ & & \\
\hline
\end{tabular}




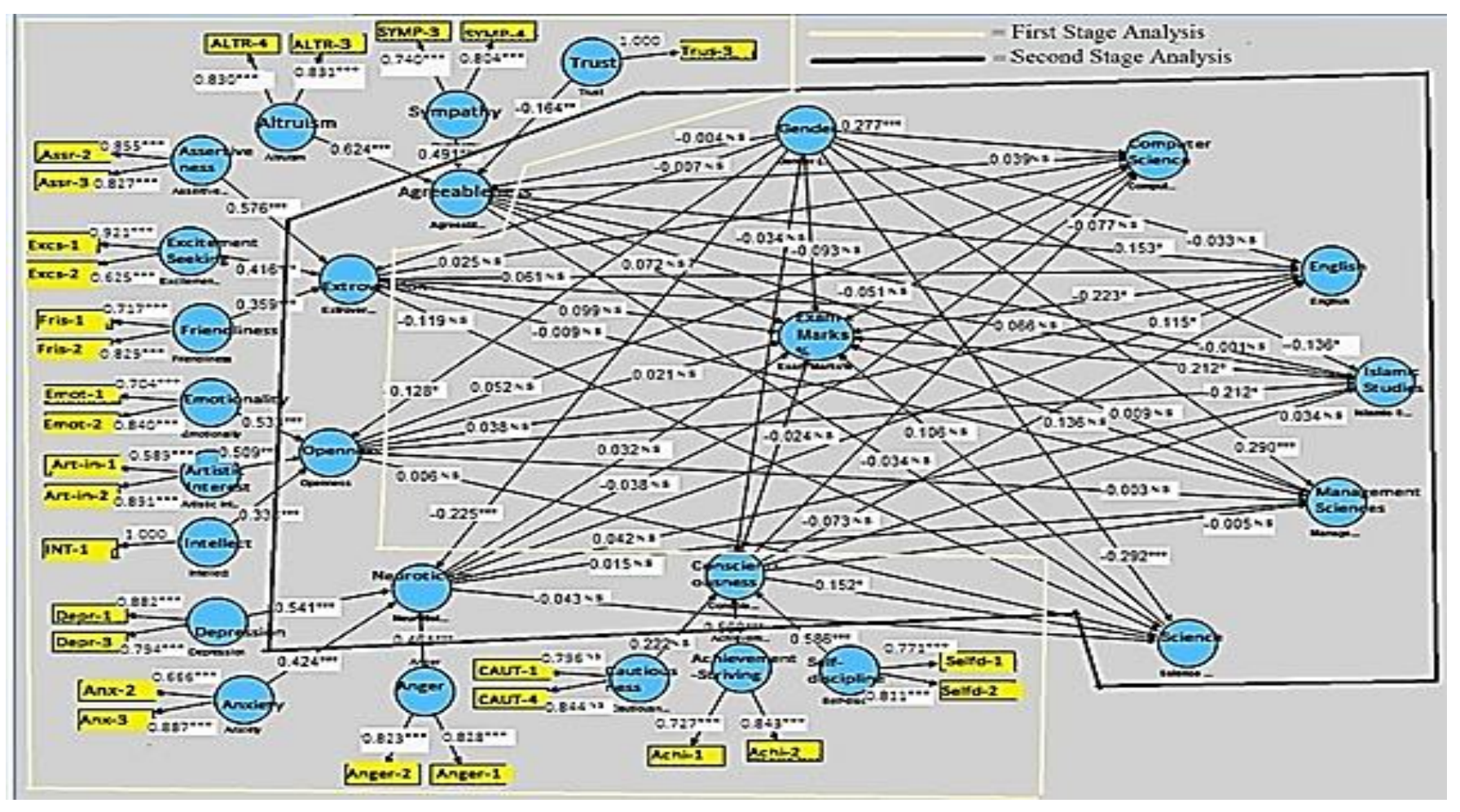

Figure No. 2: First and Second Stage SEM Analysis

Table No.2 shows the discriminant validity analysis of first-order constructs. The interrelationships of different first-order latent variables in the measurement model are lower than their square root average variance extracted (highlighted in Table No. 2). According to Fornell-Larcker Criterion, the first-order latent variables have discriminant validity (Henseler et al., 2016).

Table No. 2: Comparison of Square Root AVE Values of Latent variables (First Order) and their interrelationships

\begin{tabular}{|c|c|c|c|c|c|c|c|c|c|c|c|}
\hline & & 1 & 2 & 3 & 4 & 5 & 6 & 7 & 8 & 9 & 10 \\
\hline $\mathbf{1}$ & Achievement-Striving & 0.787 & & & & & & & & & \\
\hline 2 & Agreeableness & -0.014 & 0.626 & & & & & & & & \\
\hline 4 & Anger & 0.050 & -0.140 & -0.109 & 0.826 & & & & & & \\
\hline 5 & Anxiety & 0.161 & -0.019 & 0.028 & 0.215 & 0.784 & & & & & \\
\hline 8 & Cautiousness & 0.053 & 0.366 & 0.369 & -0.171 & -0.172 & 0.069 & 0.043 & 0.820 & & \\
\hline 9 & Conscientiousness & 0.804 & 0.032 & 0.074 & -0.015 & 0.061 & 0.373 & 0.439 & 0.289 & 0.539 & \\
\hline 10 & Depression & 0.137 & -0.119 & -0.104 & 0.204 & 0.282 & 0.076 & 0.060 & -0.118 & 0.111 & 0.839 \\
\hline 11 & Emotionality & 0.402 & 0.086 & 0.078 & 0.157 & 0.176 & 0.327 & 0.281 & 0.027 & 0.372 & 0.166 \\
\hline 12 & Excitement seeking & 0.255 & 0.058 & 0.060 & 0.103 & 0.146 & 0.309 & 0.375 & 0.000 & 0.275 & 0.103 \\
\hline 17 & Openness & 0.520 & 0.104 & 0.097 & 0.122 & 0.134 & 0.771 & 0.384 & 0.052 & 0.506 & 0.156 \\
\hline 18 & Self-discipline & 0.380 & -0.071 & -0.065 & -0.011 & 0.012 & 0.236 & 0.357 & 0.062 & 0.816 & 0.102 \\
\hline 19 & Sympathy & -0.053 & 0.806 & 0.473 & -0.161 & 0.003 & 0.081 & 0.140 & 0.214 & -0.014 & -0.071 \\
\hline 20 & Trust & 0.131 & -0.329 & -0.170 & -0.043 & 0.232 & 0.059 & 0.096 & -0.187 & 0.047 & 0.113 \\
\hline
\end{tabular}




\begin{tabular}{|c|c|c|c|c|c|c|c|c|c|c|c|}
\hline \multicolumn{12}{|c|}{$\begin{array}{l}\text { Table No. } 2 \text { (Continue .....) } \\
\text { Comparison of Square Root }\end{array}$} \\
\hline & & 11 & 12 & 13 & 14 & 15 & 16 & 17 & 18 & 19 & 20 \\
\hline 1 & Achievement-Striving & & & & & & & & & & \\
\hline 2 & Agreeableness & & & & & & & & & & \\
\hline 3 & Altruism & & & & & & & & & & \\
\hline 4 & Anger & & & & & & & & & & \\
\hline 5 & Anxiety & & & & & & & & & & \\
\hline 6 & Artistic Interest & & & & & & & & & & \\
\hline 7 & Assertiveness & & & & & & & & & & \\
\hline 8 & Cautiousness & & & & & & & & & & \\
\hline 9 & Conscientiousness & & & & & & & & & & \\
\hline 10 & Depression & & & & & & & & & & \\
\hline 11 & Emotionality & 0.775 & & & & & & & & & \\
\hline 12 & Excitement seeking & 0.246 & 0.787 & & & & & & & & \\
\hline 13 & Extraversion & 0.352 & 0.703 & 0.587 & & & & & & & \\
\hline 14 & Friendliness & 0.245 & 0.199 & 0.620 & 0.775 & & & & & & \\
\hline 15 & Intellect & 0.210 & 0.332 & 0.310 & 0.198 & 1.000 & & & & & \\
\hline 16 & Neuroticism & 0.237 & 0.165 & 0.078 & -0.050 & 0.067 & 0.570 & & & & \\
\hline 17 & Openness & 0.771 & 0.401 & 0.496 & 0.304 & 0.582 & 0.197 & 0.588 & & & \\
\hline 18 & Self-discipline & 0.233 & 0.221 & 0.356 & 0.164 & 0.281 & 0.056 & 0.340 & 0.792 & & \\
\hline 19 & Sympathy & 0.097 & 0.047 & 0.096 & -0.012 & 0.015 & -0.112 & 0.098 & -0.054 & 0.773 & \\
\hline 20 & Trust & 0.062 & 0.016 & 0.073 & 0.032 & -0.115 & 0.140 & 0.025 & 0.024 & -0.123 & 1.000 \\
\hline
\end{tabular}

\subsection{Second-Order Path Model}

Tables No.1 and 2 show that the measurement model in first-stage analysis can be used to draw second-order latent variables of second-stage analysis. Based on item scores in different sub-traits (First order latent variables), the scores of traits (Second-order latent variables) were calculated with the help of the SmartPLS2, and later these scores were used in the second-order analysis. The discriminant validity is shown in Table No.3. This table shows that square root AVE values of latent variables are greater than the interrelationships of latent variables in the second-order path model. Hence, these latent variables have discriminant validity (Henseler et al., 2016). 
Review of Economics and Development Studies, Vol. 7 (3) 2021, 433 - 451

\begin{tabular}{|c|c|c|c|c|c|c|c|c|c|c|c|c|c|}
\hline \multicolumn{14}{|c|}{ Table No.3 } \\
\hline \multicolumn{14}{|c|}{ Comparison of Square Root AVE Values of Latent variables (Second Order) and their interrelationships } \\
\hline & & 1 & 2 & 3 & 4 & 5 & 6 & 7 & 8 & 9 & 10 & 11 & 12 \\
\hline 1 & Agreeablen-ess & 1.000 & & & & & & & & & & & \\
\hline 2 & $\begin{array}{l}\text { Computer } \\
\text { Science }\end{array}$ & 0.048 & 1.000 & & & & & & & & & & \\
\hline 3 & $\begin{array}{l}\text { Conscientiou- } \\
\text { sness }\end{array}$ & 0.032 & -0.050 & 1.000 & & & & & & & & & \\
\hline 4 & English & 0.143 & -0.319 & -0.010 & 1.000 & & & & & & & & \\
\hline 5 & Extraversion & 0.063 & 0.013 & 0.461 & 0.065 & 1.000 & & & & & & & \\
\hline 6 & Islamic Studies & -0.023 & -0.111 & -0.035 & -0.151 & -0.019 & 1.000 & & & & & & \\
\hline 7 & Male & -0.004 & 0.282 & -0.034 & -0.062 & -0.007 & -0.120 & 1.000 & & & & & \\
\hline 8 & $\begin{array}{l}\text { Management } \\
\text { Sciences }\end{array}$ & -0.013 & -0.148 & -0.020 & -0.202 & -0.014 & -0.070 & 0.287 & 1.000 & & & & \\
\hline 9 & $\begin{array}{l}\text { Marks } \\
\text { (Percentage) }\end{array}$ & 0.032 & -0.079 & 0.046 & -0.282 & 0.079 & 0.233 & -0.120 & 0.122 & 1.000 & & & \\
\hline 10 & Neuroticism & -0.137 & -0.100 & 0.079 & 0.108 & 0.078 & 0.039 & -0.225 & -0.051 & 0.038 & 1.000 & & \\
\hline 11 & Openness & 0.104 & -0.014 & 0.506 & 0.074 & 0.496 & -0.137 & -0.128 & -0.046 & 0.038 & 0.197 & 1.000 & \\
\hline 12 & Science math & -0.029 & -0.303 & 0.105 & -0.414 & -0.050 & -0.144 & -0.288 & -0.192 & 0.176 & 0.031 & 0.049 & 1.000 \\
\hline
\end{tabular}

The significance of different postulated paths tested in second-order path analysis is shown in Table No. 4 and Figure No. 2. The relationships of gender with academic programs were significant. The gender male has a significant positive relationship with computer sciences, negative relationship with Islamic studies, positive relationship with management sciences, and negative relationship with science math academic programs. The variable gender has a significant impact on personality traits as well. The male gender was negatively associated with Neuroticism and positively linked with Openness personality traits.

The relationships of different academic programs with personality traits were significant as well. Table No.4 shows that the Agreeableness personality trait is positively related to English discipline, the Conscientiousness trait has a positive relationship with mathematics discipline, Neuroticism trait has a positive association with English program of study, and Openness trait has a positive relationship with Islamic studies. Concerning the relationship between personality traits and academic performance, the personality traits were not found to directly relate to academic grades. However, the likelihood of academic performance varies in different academic programs. The students of the English program of study have a probability of the lowest academic performance as compared to students of Islamic studies who have likelihood to show the highest academic performance. 
Review of Economics and Development Studies, Vol. 7 (3) 2021, 433 - 451

\begin{tabular}{|c|c|c|c|c|c|}
\hline \multicolumn{6}{|l|}{$\begin{array}{l}\text { Table No. } 4 \\
\text { Second-Order Path Coefficients }\end{array}$} \\
\hline Hypothesized Paths & $\begin{array}{l}\text { Original } \\
\text { Sample }\end{array}$ & $\begin{array}{l}\text { Sample } \\
\text { Mean }\end{array}$ & $\begin{array}{l}\text { Standard } \\
\text { Deviation }\end{array}$ & T Statistics & P Values \\
\hline \multicolumn{6}{|l|}{ Gender -> Personality Traits } \\
\hline Male -> Agreeableness & -0.004 & -0.005 & 0.053 & 0.074 & 0.941 \\
\hline Male -> Conscientiousness & -0.034 & -0.035 & 0.059 & 0.584 & 0.560 \\
\hline Male -> Extraversion & -0.007 & -0.005 & 0.064 & 0.105 & 0.917 \\
\hline Male -> Neuroticism & -0.225 & -0.224 & 0.058 & 3.864 & 0.000 \\
\hline Male -> Openness & -0.128 & -0.125 & 0.059 & 2.163 & 0.031 \\
\hline \multicolumn{6}{|l|}{ Gender -> Programs of Study } \\
\hline Male -> Computer Science & 0.277 & 0.280 & 0.066 & 4.224 & 0.000 \\
\hline Male -> English & -0.033 & -0.035 & 0.059 & 0.557 & 0.578 \\
\hline Male -> Islamic Studies & -0.136 & -0.135 & 0.042 & 3.279 & 0.001 \\
\hline Male -> Management Sciences & 0.290 & 0.286 & 0.060 & 4.807 & 0.000 \\
\hline Male -> Science math & -0.292 & -0.289 & 0.053 & 5.567 & 0.000 \\
\hline \multicolumn{6}{|l|}{ Personality Traits -> Programs of Study } \\
\hline \multicolumn{6}{|l|}{ Agreeableness -> Programs of Study } \\
\hline Agreeableness -> Computer Science & 0.039 & 0.041 & 0.053 & 0.748 & 0.455 \\
\hline Agreeableness -> English & 0.153 & 0.155 & 0.058 & 2.662 & 0.008 \\
\hline Agreeableness -> Islamic Studies & -0.001 & -0.004 & 0.057 & 0.018 & 0.986 \\
\hline Agreeableness -> Management Sciences & -0.009 & -0.013 & 0.064 & 0.144 & 0.886 \\
\hline Agreeableness -> Science math & -0.034 & -0.033 & 0.060 & 0.574 & 0.566 \\
\hline \multicolumn{6}{|l|}{ Conscientiousness -> Programs of Study } \\
\hline Conscientiousness -> Computer Science & -0.077 & -0.077 & 0.060 & 1.286 & 0.199 \\
\hline Conscientiousness -> English & -0.073 & -0.074 & 0.079 & 0.921 & 0.357 \\
\hline Conscientiousness -> Islamic Studies & 0.034 & 0.034 & 0.075 & 0.457 & 0.648 \\
\hline Conscientiousness -> Management Sciences & -0.005 & -0.003 & 0.065 & 0.074 & 0.941 \\
\hline Conscientiousness -> Science math & 0.152 & 0.154 & 0.065 & 2.329 & 0.020 \\
\hline \multicolumn{6}{|l|}{ Extraversion -> Programs of Study } \\
\hline Extraversion -> Computer Science & 0.025 & 0.021 & 0.070 & 0.350 & 0.727 \\
\hline Extraversion -> English & 0.061 & 0.063 & 0.073 & 0.834 & 0.405 \\
\hline Extraversion -> Islamic Studies & 0.066 & 0.066 & 0.044 & 1.496 & 0.135 \\
\hline Extraversion -> Management Sciences & -0.009 & -0.010 & 0.079 & 0.115 & 0.909 \\
\hline Extraversion $->$ Science math & -0.119 & -0.120 & 0.062 & 1.911 & 0.056 \\
\hline \multicolumn{6}{|l|}{ Neuroticism -> Programs of Study } \\
\hline Neuroticism -> Computer Science & -0.038 & -0.035 & 0.056 & 0.684 & 0.494 \\
\hline Neuroticism -> English & 0.115 & 0.116 & 0.055 & 2.074 & 0.038 \\
\hline Neuroticism -> Islamic Studies & 0.042 & 0.039 & 0.049 & 0.870 & 0.384 \\
\hline Neuroticism -> Management Sciences & 0.015 & 0.011 & 0.074 & 0.201 & 0.841 \\
\hline Neuroticism -> Science math & -0.043 & -0.043 & 0.061 & 0.708 & 0.479 \\
\hline \multicolumn{6}{|l|}{ Openness -> Programs of Study } \\
\hline Openness -> Computer Science & 0.052 & 0.058 & 0.066 & 0.789 & 0.430 \\
\hline Openness -> English & 0.038 & 0.031 & 0.073 & 0.521 & 0.603 \\
\hline Openness -> Islamic Studies & -0.212 & -0.209 & 0.080 & 2.662 & 0.008 \\
\hline Openness -> Management Sciences & -0.003 & -0.001 & 0.059 & 0.058 & 0.954 \\
\hline
\end{tabular}


Review of Economics and Development Studies, Vol. 7 (3) 2021, 433 - 451

\begin{tabular}{|l|l|l|l|l|l|}
\hline Openness -> Science math & 0.006 & 0.006 & 0.071 & 0.084 & \\
\hline Gender -> Marks (Percentage) & & & & & \\
\hline Male -> Marks (Percentage) & -0.093 & -0.093 & 0.071 & 1.301 \\
\hline Personality Traits -> Marks (Percentage) & & & & 0.193 \\
\hline Agreeableness -> Marks (Percentage) & 0.072 & 0.075 & 0.057 & 1.263 & 0.371 \\
\hline Conscientiousness -> Marks (Percentage) & -0.024 & -0.025 & 0.066 & 0.207 & 0.711 \\
\hline Extraversion -> Marks (Percentage) & 0.099 & 0.101 & 0.064 & 0.539 & 0.540 \\
\hline Neuroticism -> Marks (Percentage) & 0.032 & 0.028 & 0.059 & 0.124 & 0.590 \\
\hline Openness -> Marks (Percentage) & 0.021 & 0.018 & 0.074 & 0.777 \\
\hline Program of Study -> Marks (Percentage) & & & & 0.093 \\
\hline Computer Science -> Marks (Percentage) & -0.051 & -0.056 & 0.097 & 0.531 \\
\hline English -> Marks (Percentage) & -0.223 & -0.231 & 0.102 & 2.187 & 0.596 \\
\hline Islamic Studies -> Marks (Percentage) & 0.212 & 0.208 & 0.068 & 3.094 \\
\hline Management Sciences -> Marks (Percentage) & 0.136 & 0.132 & 0.078 & 1.735 & 0.029 \\
\hline Science math -> Marks (Percentage) & 0.106 & 0.102 & 0.093 & 1.142 \\
\hline
\end{tabular}

Table No. 5 shows the total effects of predictor variables on dependent variables in the secondorder path model. Although, there were significant negative direct and total effects of male gender on students' Neuroticism and Openness to experience personality traits. However, there were no significant direct or total effects of gender on Agreeableness, Conscientiousness, and Extraversion traits. Whereas the variable gender has appeared as a significant predictor of students' academic programs. After assessing the total effects, it is inferred that male students will likely be in computer sciences, and management sciences academic programs, whereas, female students will be in Islamic studies and science math programs. Although gender has no significant direct impact on students' academic performance, the total effects of gender male on academic performance through different variables in the model were significant and negative. The personality traits have no significant direct effect or total effect on students' academic performance.

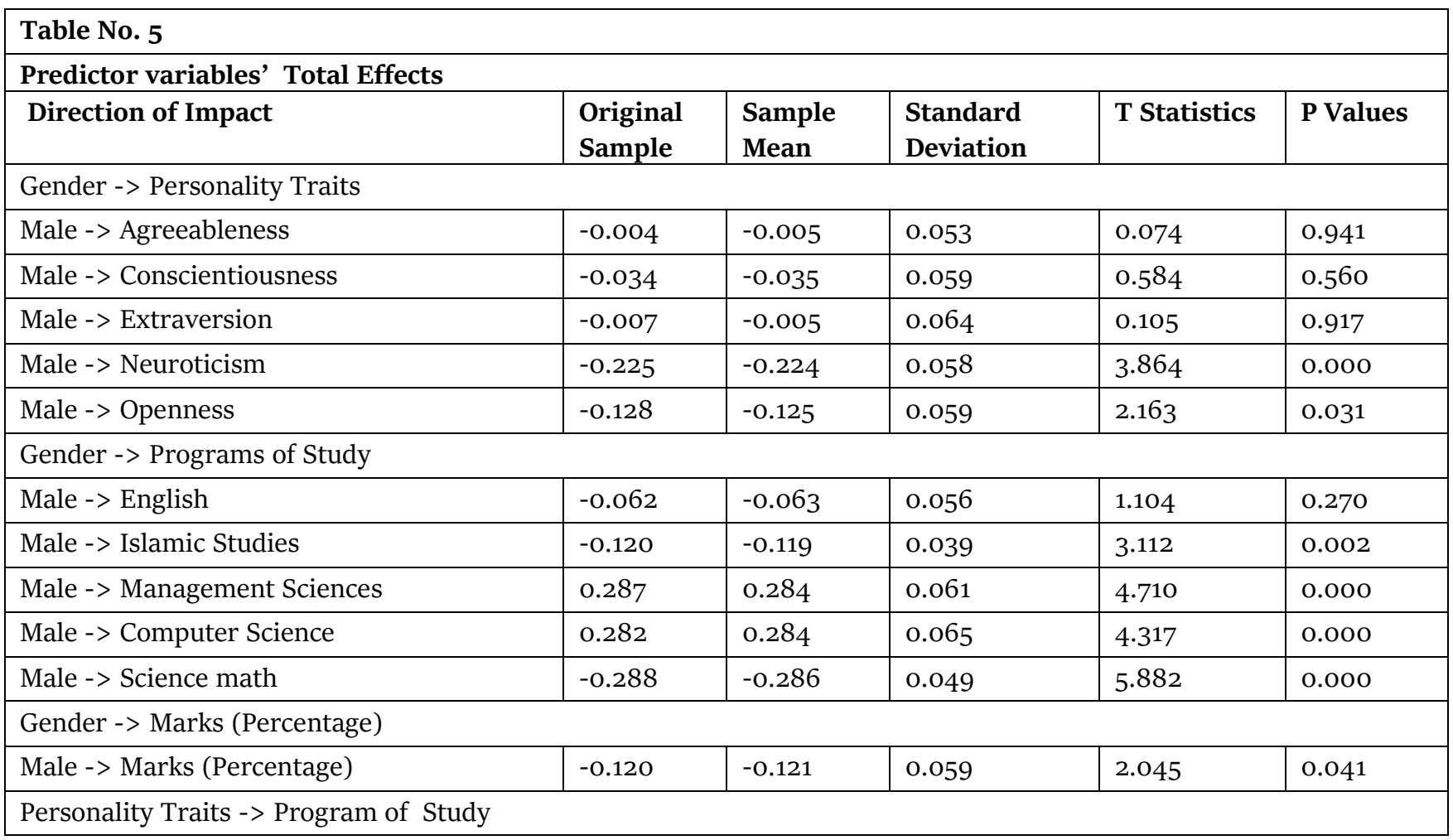


Review of Economics and Development Studies, Vol. 7 (3) 2021, 433 - 451

\begin{tabular}{|c|c|c|c|c|c|}
\hline \multicolumn{6}{|l|}{ Agreeableness -> Program of Study } \\
\hline Agreeableness -> Computer Science & 0.039 & 0.041 & 0.053 & 0.748 & 0.455 \\
\hline Agreeableness -> English & 0.153 & 0.155 & 0.058 & 2.662 & 0.008 \\
\hline Agreeableness -> Islamic Studies & -0.001 & -0.004 & 0.057 & 0.018 & 0.986 \\
\hline Agreeableness -> Management Sciences & -0.009 & -0.013 & 0.064 & 0.144 & 0.886 \\
\hline Agreeableness -> Science math & -0.034 & -0.033 & 0.060 & 0.574 & 0.566 \\
\hline \multicolumn{6}{|l|}{ Conscientiousness -> Programs of Study } \\
\hline Conscientiousness -> Computer Science & -0.077 & -0.077 & 0.060 & 1.286 & 0.199 \\
\hline Conscientiousness -> English & -0.073 & -0.074 & 0.079 & 0.921 & 0.357 \\
\hline Conscientiousness -> Islamic Studies & 0.034 & 0.034 & 0.075 & 0.457 & 0.648 \\
\hline Conscientiousness -> Management Sciences & -0.005 & -0.003 & 0.065 & 0.074 & 0.941 \\
\hline Conscientiousness -> Science math & 0.152 & 0.154 & 0.065 & 2.329 & 0.020 \\
\hline \multicolumn{6}{|l|}{ Extraversion -> Programs of Study } \\
\hline Extraversion -> Computer Science & 0.025 & 0.021 & 0.070 & 0.350 & 0.727 \\
\hline Extraversion -> English & 0.061 & 0.063 & 0.073 & 0.834 & 0.405 \\
\hline Extraversion -> Islamic Studies & 0.066 & 0.066 & 0.044 & 1.496 & 0.135 \\
\hline Extraversion -> Management Sciences & -0.009 & -0.010 & 0.079 & 0.115 & 0.909 \\
\hline Extraversion -> Science math & -0.119 & -0.120 & 0.062 & 1.911 & 0.056 \\
\hline \multicolumn{6}{|l|}{ Neuroticism -> Programs of Study } \\
\hline Neuroticism -> Computer Science & -0.038 & -0.035 & 0.056 & 0.684 & 0.494 \\
\hline Neuroticism -> English & 0.115 & 0.116 & 0.055 & 2.074 & 0.038 \\
\hline Neuroticism -> Islamic Studies & 0.042 & 0.039 & 0.049 & 0.870 & 0.384 \\
\hline Neuroticism -> Management Sciences & 0.015 & 0.011 & 0.074 & 0.201 & 0.841 \\
\hline Neuroticism -> Science math & -0.043 & -0.043 & 0.061 & 0.708 & 0.479 \\
\hline \multicolumn{6}{|l|}{ Openness -> Programs of Study } \\
\hline Openness -> Computer Science & 0.052 & 0.058 & 0.066 & 0.789 & 0.430 \\
\hline Openness -> English & 0.038 & 0.031 & 0.073 & 0.521 & 0.603 \\
\hline Openness -> Islamic Studies & -0.212 & -0.209 & 0.080 & 2.662 & 0.008 \\
\hline Openness -> Management Sciences & -0.003 & -0.001 & 0.059 & 0.058 & 0.954 \\
\hline Openness -> Science math & 0.006 & 0.006 & 0.071 & 0.084 & 0.933 \\
\hline \multicolumn{6}{|l|}{ Personality -> Marks (Percentage) } \\
\hline Agreeableness -> Marks (Percentage) & 0.031 & 0.031 & 0.058 & 0.537 & 0.592 \\
\hline Conscientiousness -> Marks (Percentage) & 0.018 & 0.018 & 0.071 & 0.259 & 0.796 \\
\hline Extraversion -> Marks (Percentage) & 0.084 & 0.088 & 0.064 & 1.320 & 0.187 \\
\hline Neuroticism -> Marks (Percentage) & 0.014 & 0.009 & 0.060 & 0.241 & 0.809 \\
\hline Openness -> Marks (Percentage) & -0.035 & -0.035 & 0.073 & 0.480 & 0.631 \\
\hline \multicolumn{6}{|l|}{ Programs of Study -> Marks (Percentage) } \\
\hline Computer Science -> Marks (Percentage) & -0.051 & -0.056 & 0.097 & 0.531 & 0.596 \\
\hline English -> Marks (Percentage) & -0.223 & -0.231 & 0.102 & 2.187 & 0.029 \\
\hline Islamic Studies -> Marks (Percentage) & 0.212 & 0.208 & 0.068 & 3.094 & 0.002 \\
\hline $\begin{array}{llll}\begin{array}{l}\text { Management } \\
\text { (Percentage) }\end{array} & \text { Sciences } & -> & \text { Marks } \\
\end{array}$ & 0.136 & 0.132 & 0.078 & 1.735 & 0.083 \\
\hline Science math -> Marks (Percentage) & 0.106 & 0.102 & 0.093 & 1.142 & 0.254 \\
\hline
\end{tabular}


Review of Economics and Development Studies, Vol. 7 (3) 2021, 433 - 451

Table No.6 shows that different predictor variables in this model explained $14 \%$ variation in students' academic performance. Likewise, this model explained the difference in a subject taking patterns up to $6.7 \%$ in computer science, $2.3 \%$ in English, $2.4 \%$ in Islamic studies, $6.3 \%$ in management sciences, and $8.6 \%$ in science math. The variable gender explained variance in Neuroticism up to $4.7 \%$ and Openness up to $1.3 \%$.

\begin{tabular}{|l|l|}
\hline \multicolumn{2}{|l|}{ Table No. 6} \\
\hline R Square Table & R Square Adjusted \\
\hline Dependent Variable & -0.004 \\
\hline Agreeableness & 0.067 \\
\hline Computer Science & -0.002 \\
\hline Conscientiousness & 0.023 \\
\hline English & -0.004 \\
\hline Extraversion & 0.024 \\
\hline Islamic Studies & 0.063 \\
\hline Management Sciences & 0.140 \\
\hline Academic Performance & 0.047 \\
\hline Neuroticism & 0.013 \\
\hline Openness & 0.086 \\
\hline Science math & \\
\hline
\end{tabular}

\section{Discussion}

The results of this study indicate the importance of gender in the selection of academic programs. The current study validates earlier findings that variable gender impacts students' subject preferences (Francis, 2000; Lörz et al., 2011). It is affirmed in this study that Pakistani male university students have stereotypical male subject choices. This study has established that computer sciences and management sciences academic programs are related to the male gender. This trend is congruent with the literature. Computer sciences and management sciences subjects are traditionally considered masculine subjects (Gautam, 2015; UNECE, 2019). Whereas Pakistani female university students' subject preferences somewhat differ from female stereotype subject preferences. Although science math subjects were traditionally considered male-gendered subjects (Cavaglia et al., 2021), this study found fewer males in science math academic programs than females. The trend of an increase in female students' interest in science math subjects is obvious in recent studies in many countries (UNECE, 2019). Likewise, the Pakistani university female students' preference for Islamic studies program affirms their stereotype female subject choice because females are traditionally interested in humanities and arts subjects (van der Vleuten et al., 2016), The female students' increased interest in science math subjects might be a result of social acceptance of females as scientists and social rejection of gendered ability beliefs (Dom \& Yi, 2018). However, the role of external factors in subject choices in Asian societies cannot be overlooked. The family especially the father, availability of institution, location, and availability of hostel facilities also play a significant role in subject choices in Asian societies (Alwedinani, 2016; Gautam, 2015).

For gender and personality traits, it is reiterated in this study that gender impacts personality traits. The Pakistani university students' male gender is found to have negative relationships with Neuroticism and Openness personality traits. The gender differences in personality traits found in this study are consistent with past studies. The literature supports findings that female gender is associated with higher Neuroticism (Chapman et al., 2007; Kim et al., 2016; Rahmani \& Lavasani, 2012; Weisberg 
Review of Economics and Development Studies, Vol. 7 (3) 2021, 433 - 451

et al., 2011) and Openness to experience (Busato et al., 1998; Kim et al., 2016). It means that there are similar gender roles and gender perceptions in eastern and western societies, which generate similar personality traits (Costa Jr. et al., 2001; Schmitt et al., 2017; Yousaf Zai \& Jan, 2019).

The current study affirms that students' personality traits are associated with their academic programs. The Pakistani university students enrolled in English literature have higher Agreeableness and Neuroticism traits. Whereas Pakistani university students of science math programs appeared to have higher Conscientiousness. These findings validate previous studies. Vedel (2016) found students of arts and humanities to have higher Neuroticism and Agreeableness traits as compared to science students who appeared to have higher Conscientiousness. Interestingly, the Openness to experience trait is associated with students of Islamic studies. This finding is not contradictory to earlier findings. It is worth noting that the Islamic studies discipline in this study has appeared as a female-gendered subject. Traditionally, females are found to have higher Openness to experience (Busato et al., 1998; Kim et al., 2016).

Although, the total indirect associations of Pakistani University students' male gender with their academic performance through different variables in the model is significant and negative. Unexpectedly, the Pakistani university students' academic performance is not directly related to their gender. The academic performance of female students is found higher than the male students. This finding affirms the results of previous studies (Cavaglia et al., 2021). The academic performance of female students has improved over the years as compared to the decrease in male students' academic performance (Hdii \& Fagroud, 2018; Sparks-Wallace, 2007).

Surprisingly, the Pakistani university students' personality traits did not have a significant direct or total effect on their academic performance. This finding is incongruent with the results of several previous studies. The effect of students' personality traits on their academic performance is noticeable in earlier findings (Blickle, 1996; O'Connor \& Paunonen, 2007). The literature supports that Conscientiousness, Agreeableness, and Openness to experience personality traits are positive predictors of students' academic performance (Poropat, 2009) as compared to Extraversion and Neuroticism that are negative predictors of academic performance (Komarraju et al., 2011; O'Connor \& Paunonen, 2007). This inconsistency may be because Pakistani university students choose subjects because of external factors rather than internal motivations, therefore their personality traits are not reflected in their academic endeavours and academic outcomes.

As postulated, there are significant associations between academic programs and students' academic performance. The academic program of English literature has a negative association with students' academic performance as compared to the academic program of Islamic Studies which has a significant positive relationship with students' academic performance. The probable causes of these findings are students' gender and their personality traits. Although English literature is not significantly related to gender, the sampled Pakistani English literature students have higher Neuroticism. The negative impact of Neuroticism on students' academic performance is consistently reported in previous studies (Komarraju et al., 2011). Therefore, the presence of high Neuroticism in sampled Pakistani students of English literature is the probable cause for their low academic performance as compared to students of other academic programs. Likewise, the sampled Pakistani students of Islamic studies are mostly females and they have higher Openness to experience. It is evident in literature that females might have better academic performance than males (Hdii \& Fagroud, 2018; Sparks-Wallace, 2007), and Openness to experience trait has a positive impact on students' academic performance (Cavaglia et al., 2021; Poropat, 2009). Therefore, it may be recognized that the Islamic studies program of study has 
Review of Economics and Development Studies, Vol. 7 (3) 2021, 433 - 451

a positive relationship with academic performance because it is a female-gendered subject and the students in this program of study have high Openness to experience. Another possible explanation of higher academic performance in students of Islamic studies may be the use of Urdu as the medium of instruction and examination in this program. The remaining all academic programs have an English medium of instruction. English is a foreign language for students and Urdu is the national language of Pakistan.

\section{Conclusion}

Academic performance depends on students' learning behaviours in a learning situation. However, students' gender, personality, and academic programs are the few important attributes that define their learning behaviours. However, these attributes have gender differences because of socialization. The gendered stereotype perceptions of social roles and misconceptions of abilities promote stereotype personality traits and stereotype subject choices in students that consequently impact academic performance. Neuroticism and Openness to experience traits are obvious in sampled female students because of gendered stereotype social development. However, there is a change to a certain degree in female's subject choices in this study and they chose science subjects. Although different personality traits are related to students' subject choices, personality traits do not seem to be related to academic performance. It shows that students have similar learning behaviours irrespective of their personalities.

\section{Recommendations}

This study is limited to university students, however, this phenomenon should be studied at school levels. There is a need to understand the phenomenon of gendered personalities, subject choices, and academic performance by conducting qualitative studies in Pakistan. It will be pertinent to understand the role of different social institutions in the inculcation of gendered stereotypes in individuals. Further research is needed to understand the way stereotype gendered personalities and subject choices impact the larger social landscape. It is recommended that future studies should focus on the home environment, and social environment to understand how gendered stereotypes flourish in societies. It is of utmost need that Neurotic students should be identified and assisted to overcome the negative aspects of their personalities.

The study recommends that students' academic performance can be improved by addressing stereotype gender perceptions, stereotype personalities, and traditional subject choices. There is a need to involve social media, parents, and students to reduce stereotypes in society. Society should promote anti-stereotype views among individuals so that they can learn with their full potential as human beings.

Interventions should be designed to reduce and eliminate gendered environments at home, school, and community. The schools, colleges, universities, and societies should encourage both genders to opt for subjects based on their interests and talents.

\section{References}

Akter, S., Fosso Wamba, S., \& Dewan, S. (2017). Why PLS-SEM is suitable for complex modeling? An empirical illustration in Big Data Analytics Quality. Production Planning and Control, 28(11-12), 1011-1021. Retrieved from https://ro.uow.edu.au/cgi/viewcontent.cgi?referer= \&httpsredir $=1 \&$ article $=2248 \&$ context $=$ buspapers

Ali, R., Hussain, I., \& Rahmani, S. H. (2019). How Socioeconomic Classes Influence Academic Grades of Elementary School Students? Defining Mediation Role of School Backgrounds and 
Review of Economics and Development Studies, Vol. 7 (3) 2021, 433 - 451

Cognitive Processing Strategies. Journal of Educational Research (1027-9776), 22(2), 201227.

Alwedinani, J. (2016). Gender and Subject Choice in Higher Education in Saudi Arabia. (PhD Thesis), University of York. Retrieved from https://etheses.whiterose.ac.uk/15372/

Andersen, S. C., Gensowski, M., Ludeke, S. G., \& John, O. P. (2020). A stable relationship between personality and academic performance from childhood through adolescence. An original study and replication in hundred-thousand-person samples. Journal of Personality, 88(5), 925-939. doi: 10.1111/jopy.12538

Anderson, J. C., \& Gerbing, D. W. (1988). Structural equation modeling in practice: A review and recommended two-step approach. Psychological Bulletin, 103(3), 411-423. doi:10.1037/oo33-2909.103.3.411

Balsamo, M., Lauriola, M., \& Saggino, A. (2012). Personality and College Major Choice: Which Come First? Psychology, 3(5), 399-405. doi: 10.4236/psych.2012.35056

Becker, J.-M., Klein, K., \& Wetzels, M. (2017). Hierarchical Latent Variable Models in PLS-SEM: Guidelines for Using Reflective-Formative Type Models. Long Range Planning, 45, 359-394. Retrieved from https://www.researchgate.net

Blickle, G. (1996). Personality traits, learning stratigies, and performance. European Journal of Personality, 10(5), 337-352. doi:10.1002/(sici)1099-0984(199612)10:5<337::aidper258>3.0.co;2-7

Busato, V. V., Prins, F. J., Elshout, J. J., \& Hamaker, C. (1998). The relation between learning styles, the Big Five personality traits and achievement motivation in higher education. $\begin{array}{llll}\text { Personality and Individual } & \text { Differences, }\end{array}$ doi:http://dx.doi.org/10.1016/So191-8869(98)oo112-3

Cachia, M., Lynam, S., \& Stock, R. (2018). Academic success: Is it just about the grades? Higher Education Pedagogies, 3(1), 434-439. doi:10.1080/23752696.2018.1462096

Cavaglia, C., Machin, S., McNally, S., \& Ruiz-Valenzuela, J. (2021). Gender, achievement, and subject choice in English education. Oxford Review of Economic Policy, 36(4), 816-835. doi:10.1093/oxrep/graao50

Chapman, B. P., Duberstein, P. R., Sörensen, S., \& Lyness, J. M. (2007). Gender Differences in Five Factor Model Personality Traits in an Elderly Cohort: Extension of Robust and Surprising Findings to an Older Generation. Personality and Individual Differences, 43(6), 1594-1603. doi:10.1016/j.paid.2007.04.028

Costa Jr., P. T., Terracciano, A., \& McCrae, R. R. (2001). Gender Differences in Personality Traits Across Cultures: Robust and Surprising Findings Journal of Perf'onality and Social Psydwlog , 81(2), 222-231. Retrieved from https://www.cin.ufpe.br/ ssj/Genderdifferences\%2oin\%2opersonality\%2otraits\%2oacro ss\%20 cultures\%2oRobust\%20andsurprising\%2ofindings.pdf

Crowther, P., \& Briant, S. (2021). Predicting Academic Success: A Longitudinal Study of University Design Students. International Journal of Art \& Design Education, 40(1), 20-34. doi: 10.1111/jade.12329

Dom, V., \& Yi, G. (2018). Gender and Subject Choice: An Empirical Study on Undergraduate Students' Majors in Phnom Penh. Asian Journal of Social Sciences \& Humanities, 7(1), 4047. Retrieved from https://files.eric.ed.gov/fulltext/ED581226.pdf

Francis, B. (2000). The Gendered Subject: Students' Subject Preferences and Discussions of Gender and Subject Ability. Oxford Review of Education, 26(1), 35-48. Retrieved from http://www.jstor.org/stable/1050948

Gasteen, A. (2019). Gender and STEM Subject Choice. International Journal of Population Data Science, 4(3). doi:10.23889/ijpds.v4i3.1328 
Review of Economics and Development Studies, Vol. 7 (3) 2021, 433 - 451

Gautam, M. (2015). Gender, Subject Choice and Higher Education in India: Exploring 'Choices' and 'Constraints' of Women Students. Contemporary Education Dialogue, 12(1), 31-58. doi:10.1177/o973184914556865

Gonzalez, A. M., Oh, H. J. J., \& Baron, A. S. (2020). The Hidden Classroom: How Gender Stereotypes Impact Academic Achievement. , 10.1017/9781108235532(18), 295-314. doi:10.1017/9781108235532.018. In F. C. Worrell, T. L. Hughes, \& D. D. Dixson (Eds.), The Cambridge Handbook of Applied School Psychology: Cambridge University Press.

Hair, J., F., Hult, G., Tomas, M., Ringle, C., M., \& Sarstedt, M. (2014). Primer on Partial Least Squares Structural Equation Modeling (PLS-SEM). California, USA: SAGE Publications, Inc.

Hair, J. F., Risher, J. J., Sarstedt, M., \& Ringle, C. M. (2019). When to use and how to report the results of PLS-SEM. European Business Review, 31(1), 2-24. doi:10.1108/EBR-11-2018-0203

Hdii, S., \& Fagroud, M. (2018). The Effect of Gender on University Students' School Performance: The Case of the National School of Agriculture in Meknes, Morocco. KULTŪRA IR VISUOMENĖ. Socialinių tyrimų žurnalas, 9(1), 67-78. doi:https://dx.doi.org/10.7220/23358777.9.1.4

Henseler, J., Hubona, G., \& Ray, P. A. (2016). Using PLS path modeling in new technology research:updated guidelines. Industrial Management \& Data Systems, 116(1), 2-10. doi:DOI 10.1108/IMDS-09-2015-0382

Islam, K., \& Asadullah, M. (2018). Gender stereotypes and education: A comparative content analysis of Malaysian, Indonesian, Pakistani and Bangladeshi school textbooks. PLoS ONE, 13(1), e0190807. doi:https://doi.org/10.1371/journal.pone.0190807

Johnson, J. A. (2014). Measuring thirty facets of the Five Factor Model with a 120-item public domain inventory: Development of the IPIP-NEO-120. Journal of Research in Personality, 51, 78-89. doi:https://doi.org/10.1016/j.jrp.2014.05.003

Kim, S. E., Kim, H.-N., Cho, J., Kwon, M.-J., Chang, Y., Ryu, S., . . . Kim, H.-L. (2016). Direct and Indirect Effects of Five Factor Personality and Gender on Depressive Symptoms Mediated by Perceived Stress. PLoS ONE, 11(4), e0154140. doi:10.1371/journal.pone.0154140

Komarraju, M., Karau, S. J., Schmeck, R. R., \& Avdic, A. (2011). The Big Five personality traits, learning styles, and academic achievement. Personality and Individual Differences, 51(4), 472-477. doi:http://dx.doi.org/10.1016/j.paid.2011.04.019

Korpershoek, H., Kuyper, H., \& van der Werf, M. P. C. (2012). The Role of Personality in Relation to Gender Differences in School Subject Choices in Pre-University Education. Sex Roles, 67, 630-645. doi:DOI 10.1007/s11199-012-0222-7

Kpolovie, P. J. (2017). Intelligence and Academic Achievement: A Longitudinal Survey. International Journal of Health and Psychology Research, 5(1), 33-70. doi:https://www.eajournals.org/wp-content/uploads/Intelligence-and-Academic-

Achievement-A-Longitudinal-Survey.pdf

Lamas, H. A. (2015). School Performance. Propósitos y Representaciones, 3(1), 313-386. doi:http://dx.doi.org/10.20511/pyr2015.v3n1.74

Lörz, M., Schindler, S., \& Walter, J. G. (2011). Gender inequalities in higher education: extent, development and mechanisms of gender differences in enrolment and field of study choice. Irish Educational Studies, 30(2), 179-198. doi:10.1080/03323315.2011.569139

Ma, X. (2001). Stability of School Academic Performance across Subject Areas. Journal of Educational Measurement, 38(1), 1-18. Retrieved from http://www.jstor.org/stable/1435436

MacCann, C., Jiang, Y., Brown, L. E. R., Double, K. S., Bucich, M., \& Minbashian, A. (2020). Emotional intelligence predicts academic performance: A meta-analysis. Psychological Bulletin, 146(2), 150-186. doi:10.1037/buloooo219 
Review of Economics and Development Studies, Vol. 7 (3) 2021, 433 - 451

Matz, R. L., Koester, B. P., Fiorini, S., Grom, G., Shepard, L., Stangor, C. G., . . . McKay, T. A. (2017). Patterns of Gendered Performance Differences in Large Introductory Courses at Five Research Universities. AERA Open, 3(4), 1-12. doi:10.1177/2332858417743754

McDill, E. L., Meyers, E. D., \& Rigsby, L. C. (1967). Institutional Effects on the Academic Behavior of High School Students. Sociology of Education, 40(3), 181-199. doi:10.2307/2112074

Nortvedt, G. A., Gustafsson, J., \& Lehre, A. W. (2016). The Importance of Instructional Quality for the Relation Between Achievement in Reading and Mathematics. In N. T \& G. JE (Eds.), Teacher Quality, Instructional Quality and Student Outcomes. IEA Research for Education (A Series of In-depth Analyses Based on Data of the International Association for the Evaluation of Educational Achievement (IEA)) (Vol. 2, pp. 97-113). Cham: Springer.

O’Connor, M. C., \& Paunonen, S. V. (2007). Big Five personality predictors of post-secondary academic performance. Personality and Individual Differences, 43(5), 971-990. doi:http://dx.doi.org/10.1016/j.paid.2007.03.017

Pelch, M. (2018). Gendered differences in academic emotions and their implications for student success in STEM. International Journal of STEM Education, 5(1), 33. doi:10.1186/s40594018-0130-7

Poropat, A. E. (2009). A meta-analysis of the five-factor model of personality and academic performance. Psychological Bulletin, 135(2), 322-338. doi:10.1037/ao014996

PSLM, Pakistan Social and Living Standards Measurement. (2021). PSLM - 2019-20 Pakistan Social and Living Standards Measurement Survey (2019-20) District Level Survey. Retrieved from Islamabad: https://www.pbs.gov.pk

Rahmani, S., \& Lavasani, M. G. (2012). Gender differences in five factor model of personality and sensation seeking Procedia - Social and Behavioral Sciences, 2012(46), 2906 - 2911. doi:10.1016/j.sbspro.2012.05.587

Ringle, C. M., Sarstedt, M., \& Straub, D. W. (2012). Editor's Comments: A Critical Look at the Use of PLS-SEM in "MIS Quarterly". MIS Quarterly, 36(1), iii-xiv. doi:10.2307/41410402

Roni, S. M., Djajadikerta, H., \& Ahmad, M. A. N. (2015). PLS-SEM Approach to Second-order Factor of Deviant Behaviour: Constructing Perceived Behavioural Control. Procedia Economics and Finance, 28, 249-253. doi:https://doi.org/10.1016/S2212-5671(15)01107-7

Schmitt, D. P., Long, A. E., McPhearson, A., O'Brien, K., Remmert, B., \& Shah, S. H. (2017). Personality and gender differences in global perspective. International Journal of Psychology, 52(S1), 45-56. doi:https://doi.org/10.1002/ijop.12265

Schommer-Aikins, M., \& Easter, M. (2008). Epistemological beliefs' contributions to study strategies of Asian Americans and European Americans. Journal of Educational Psychology, 100(4), 920-929. doi:10.1037/0022-0663.100.4.920

Shulrufa, B., Keuskampb, D., \& Brakea, D. (2010). The impact of course-taking on academic achievements a systematic review and Meta analysis. Procedia Social and Behavioral Sciences, 2010(2), 3401-3406. Retrieved from https://pdf.sciencedirectassets.com

Sparks-Wallace, O. J. (2007). A Study of Gender Differences in Academic Performance in a Rural County in Tennessee. (Master of Arts in Sociology), East Tennessee State University Johnson City, Tennessee. Retrieved from https://dc.etsu.edu/cgi/viewcontent.cgi?article=3462\& context=etd

Tehseen, S., Sajilan, S., Gadar, K., \& Ramayah, T. (2017). Assessing Cultural Orientation as a ReflectiveFormative Second Order Construct - A Recent PLS-SEM Approach. Review of Integrative Business and Economics Research, 6(2), 38-63. Retrieved from https://www.buscompress.com/uploads/3/4/9/8/34980536 /riber6-2_o3k17-077_3863.pdf

Thien, L. M. (2020). Assessing a second-order quality of school life construct using partial least 
Review of Economics and Development Studies, Vol. 7 (3) 2021, 433 - 451

squares structural equation modelling approach. International Journal of Research \& Method in Education, 43(3), 243-256. doi:10.1080/1743727X.2019.1662779

Thomson, S. (2018). Achievement at school and socioeconomic background-an educational perspective. npj Science of Learning, 3(1), 5. doi:10.1038/s41539-018-0022-0

UNECE, The United Nations Economic Commission for Europe (2019). Women outnumber men in higher education but gender stereotyped subject choices persist: Statistics. Retrieved from Geneva, Switzerland: https://unece.org/statistics/news/women-outnumber-men-highereducation-gender-stereotyped-subject-choices-persist

van der Vleuten, M., Jaspers, E., Maas, I., \& van der Lippe, T. (2016). Boys' and girls' educational choices in secondary education. The role of gender ideology. Educational Studies, 42(2), 181-200. doi:10.1080/03055698.2016.1160821

van Riel, A. C. R., Henseler, J., Kemény, I., \& Sasovova, Z. (2017). Estimating hierarchical constructs using consistent partial least squares The case of second-order composites of common factors. Industrial Management \& Data Systems, 117 (3), 459-477. doi: 10.1108/IMDS-07-2016-0286

Vedel, A. (2016). Big Five personality group differences across academic majors: A systematic review. Personality and Individual Differences, 92, 1-10. doi:10.1016/j.paid.2015.12.011

Vermunt, J., \& Vermetten, Y. (2004). Patterns in Student Learning: Relationships Between Learning Strategies, Conceptions of Learning, and Learning Orientations. Educational Psychology Review, 16(4), 359-384. doi:10.1007/s10648-004-0005-y

Watson, A. K., \& Monroe, E. E. (1990). Academic achievement: A study of relationships of IQ, communication apprehension, and teacher perception. Communication Reports, 3(1), 2836. doi:10.1080/o8934219009367498

Weinert, F. E., Schrader, F.-W., \& Helmke, A. (1989). Quality of instruction and achievement outcomes. International Journal of Educational Research, 13(8), 895-914. doi:10.1016/o883-0355(89)90072-4

Weisberg, Y. J., Deyoung, C. G., \& Hirsh, J. B. (2011). Gender Differences in Personality across the Ten Aspects of the Big Five. Frontiers in psychology, 2, 178-178. doi:10.3389/fpsyg.2011.00178

Whitehead, J. M. (1996). Sex stereotypes, gender identity and subject choice at A-level. Educational Research, 38(2), 147-160. doi:10.1080/0013188960380203

York, T. T., Gibson, C., \& Rankin, S. (2015). Defining and Measuring Academic Success. Practical Assessment, Research, and Evaluation, 20. doi:https://doi.org/10.7275/hz5x-txo3

Yousaf Zai, S. A., \& Jan, A. (2019). Gender and Regional Differences in Five Factor Personality Traits among Students at Secondary Level in Punjab, Pakistan. University of Wah Journal of Social Sciences, 2(1), 42-58. Retrieved from

https://uwjss.org.pk/downloads/v2/issue1/o20103.pdf. 